\title{
HLA-DP Antigen
}

National Cancer Institute

\section{Source}

National Cancer Institute. HLA-DP Antigen. NCI Thesaurus. Code C71274.

HLA-DP antigen is a class of glycoproteins that consist of dimers of alpha and beta subunits, which are encoded by the polymorphic human genes HLA-DPA1 and HLADPB1, respectively. This protein complex is expressed primarily in lymphoid cells and function to present antigens for CD4+ T lymphocytes. 